\title{
Hepatitis E Virus (HEV) in Imported and Domestic Camels in Saudi Arabia - A Cross-Sectional Descriptive Molecular Study
}

\section{Sherif A. El-Kafrawy}

King Abdulaziz University

Ahmed M. Hassan

King Abdulaziz University

Mai M. El-Daly

King Abdulaziz University

Mohammed Al-Hajri

Ministry of Public Health

Elmoubashar Farag

Ministry of Public Health

\section{Fatimah Ahmed Elnour}

Ministry of Public Health

Anas Khan

Ministry of Health

Ahmed M. Tolah

King Abdulaziz University

Thamir A. Alandijany

King Abdulaziz University

Noura A. Othman

King Abdulaziz University

\section{Ziad A Memish}

King Saud Medical City

Victor M. Corman

Charité - University Medicine Berlin

Christian Drosten

Charité - University Medicine Berlin

Alimuddin Zumla

University College London

Esam I. Azhar ( $\nabla$ eazhar@kau.edu.sa )

King Abdulaziz University 


\section{Research Article}

Keywords: Emerging, Zoonoses, Camels, Dromedary, Hepatitis E, Hepatitis E virus, DcHEV seroprevalence Posted Date: November 30th, 2021

DOI: https://doi.org/10.21203/rs.3.rs-1074591/v1

License: (c) (i) This work is licensed under a Creative Commons Attribution 4.0 International License. Read Full License 


\section{Abstract}

Camels gained attention since the discovery of MERS-CoV as intermediary hosts for potentially epidemic zoonotic viruses. DcHEV is a novel zoonotic pathogen associated with camel contact. This study aimed to genetically characterize DcHEV in domestic and imported camels in Saudi Arabia.

DcHEV was detected by RT-PCR in serum samples, PCR-positive samples were subjected to sequencing and phylogenetic analyses.

DcHEV was detected in $1.77 \%$ of samples with higher positivity in domestic DCs. All positive imported dromedaries were from Sudan with age declining prevalence. Domestic DcHEV sequences clustered with sequences from Kenya, Somalia, and UAE while imported sequences clustered with one DcHEV isolate from UAE and both sequences clustered away from isolates reported from Pakistan. Full-genome sequences showed 24 amino acid difference with reference sequences. Our results confirm the detection of DcHEV in domestic and imported DCs. Further investigations are needed in human and camel populations to identify DcHEV potential zoonosis threat.

\section{Research In Context}

Evidence before this study: We searched PubMed, Web of Science, and Google Scholar for studies on HEV as zoonotic infections from August $1^{\text {st }} 2020$ until August $1^{\text {st }} 2021$. We used the term HEV combined with any single other term from the following list: "humans", "animals", "camels*", "dromedaries*", "phylogeny", "Africa", "sequence", and "transmission". Recent reports of high seroprevalence HEV infection in pregnant Ethiopian women led to detection of HEV-specific antibodies in Ethiopian Dromedary camels (DCs). Several studies have demonstrated HEV antibodies in dromedary camels in Africa and the Middle East. A novel HEV genotype, the Dromedary camel hepatitis E virus (DcHEV), was first reported from the UAE in a transplant patient with chronic hepatitis and was linked to consuming camel milk and meat. Recent studies of DcHEV in DCs from UAE have shown DcHEV-specific antibodies and presence of DcHEV viral genomes. Saudi Arabia is the world's largest importer of DCs from African countries. Whilst serological studies have detected DcHEV antibodies in Saudi DCs imported from Africa, RNA evidence of presence of virus remains to be defined. Detailed molecular and phylogenetic characterization of the DcHEV circulating in DCs in the country is critically important for surveillance and evolution of human infections, and design of appropriate control and preventive measures.

\section{Added value of this study}

To our knowledge, our study of DCHEV in domestic and imported dromedary camels from Africa is the largest and most comprehensive work published to date. Domestic DCs from Saudi Arabia had higher prevalence of DcHEV RNA than imported camels from Africa. This might reflect the younger age of domestic camels where infection acquired early in life had been cleared DcHEV. Phylogenetic analysis of the RdRp sequences identified infection with HEV genotype 7. Compared to the HEV genotype 7 sequence KJ496144, the two full genome sequences from this study showed 242 nucleotide variations including 24 
nonsynonymous mutations leading to the amino acid changes. Sequences from domestic camels formed a separate cluster compared to published sequences from Kenya, Somalia, and UAE. The sequences of imported camels clustered with one DCHEV isolate previously reported from UAE indicating a possible common source of animal infection. Both domestic and imported sequences clustered away from isolates reported from Pakistan. Further investigation is needed to clarify the significance of these differences.

\section{Implications of all the available evidence}

Our results provide evidence for the presence of DcHEV in both domestic and imported camels from Africa with evolving genomic changes. Any change in transmissibility and virulence of DcHEV in DCs would affect the risks of spread to humans and its potential impact on national and regional public health security. Further surveillance studies are required to identify the role of these DcHEV amino acid changes in the course of the disease and viral transmission. Further clinical, serological, molecular and epidemiology investigations of DcHEV in human populations in the Middle East and Africa are needed to define and monitor the potential threat of DcHEV to Middle eastern and global public health security in light of 10 million pilgrims who visit Saudi Arabia every year.

\section{Introduction}

Approximately $60 \%$ of all human pathogens and $75 \%$ of emerging infectious diseases are zoonotic (of animal origin). Contact with Dromedary camels (DCs) or their products was identified as major risk factor for zoonotic spillover to humans of the novel lethal coronavirus, Middle East Respiratory Syndrome Coronavirus (MERS-CoV) [1-4]. MERS-CoV remains in the WHO Blueprint list of priority pathogens. Since the first discovery of MERS-CoV, increasing attention is being focused on dromedary camels (DCs) as intermediary hosts for novel zoonotic viruses with epidemic potential.

Several recent reports of possible transmission of Hepatitis E Virus (HEV) to humans through consumption of animal products and/or frequent contact with animals have led to the acknowledgement of HEV as an important emerging zoonotic pathogen of humans of global public health significance [5]. $\mathrm{HEV}$ is a member of the family Hepeviridae, genus Orthohepevirus $A$. The virus is a common cause of acute viral hepatitis in humans, causing up to 20 million human infections annually. HEV is one of the leading causes of illness in the developing world, is endemic in Central Asia, with outbreaks reported from the Middle East and Central America. HEV has relatively high mortality rates among pregnant women and young children. Chronic HEV infection can cause more severe disease in immunocompromised individuals, such as people living with HIV, organ transplants and other immunosuppressive states.

There are four main genotypes, HEV1, HEV2, HEV3 and HEV4 [6]. HEV1 and HEV2 infect only humans. $\mathrm{HEV}$ is shed in the stools of infected persons and is transmitted in areas with poor sanitation through contaminated water supplies. In areas with good sanitation and pure water supplies, animals are a major source of HEV infection to humans, human to human transmission is also reported through blood transfusion [7]. HEV has been identified in a wide range of mammals including pigs, boar, deer, rodents, 
ferrets, rabbits, mongoose, bats, cattle, sheep, foxes, minks, and horses [8] [9-11]. HEV3 and HEV4 circulate in several animals such as pigs, wild boar, deer, other mammals and infect humans through consumption of contaminated raw or undercooked pork or game meat [12]. Several studies have reported serological evidence of HEV in blood donors, hemodialysis patients and blood transfusion recipients [1316]. A novel HEV genotype, named Dromedary camel hepatitis E virus (DcHEV), was initially detected in Dromedary camels (DCs) in the Middle East in 2014 [17]. DcHEV was first reported to cause chronic hepatitis in a transplant patient in UAE who regularly consumed camel milk and meat [18]. Recent studies from UAE have confirmed the presence of DcHEV-specific viral genomes in DCs [19].

In Saudi Arabia and other Middle Eastern communities, camels serve considerable social and economic roles since they are used as domestic pets, reared for their milk and meat, racing and beauty contests. Saudi Arabia is the world's largest importer of DCs from African countries, while countries of the horn of Africa are the main exporter of camels to the Arabian Peninsula [2, 20]. In Saudi Arabia, we recently showed that the prevalence of DcHEV antibodies in imported camels is slightly higher than in camels imported from Africa [21]. Identifying DcHEV genotypes and performing detailed molecular and phylogenetic characterization of the virus in DCs is critical for surveillance, understanding the evolution of human infections and designing appropriate control and preventive measures. Therefore, we performed thismolecular and phylogenetic analyses of DcHEV in domestic and imported camels in Saudi Arabia.

\section{Materials And Methods}

\section{Ethical approval}

Ethical permission to conduct this study was obtained from the Directorate of Agriculture, Ministry of Environment, Water and Agriculture, Jeddah, Saudi Arabia. The study was approved by the Unit of Biomedical Ethics, King Abdulaziz University Hospital, (Approval number 16-121).

\section{Dromedary Camels}

Two cohorts of DCs were enrolled in this study. The first cohort comprised DCs imported into Saudi Arabia on incoming ships from Sudan or Djibouti at the Sea Port of Jeddah. Samples were collected over a period of 2 years from 2017 to 2019. The second cohort comprised local DCs in Jeddah, Saudi Arabia and were collected from three camel farms and from an abattoir in the city before camels were slaughtered. The study was approved by the Unit of Biomedical Ethics, King Abdulaziz University Hospital, (Approval number 16-121), all methods were carried out in accordance with relevant guidelines and regulations. The study is reported in accordance with ARRIVE guidelines.

\section{Sample collection}

Jugular blood samples were collected from both cohorts of DCs. The blood samples were left to clot and then centrifuged, serum obtained and stored at $-80^{\circ} \mathrm{C}$ till testing. 
Serum samples were pooled in 9 samples per pool. RNA was extracted from serum pools using the Qiaquick viral RNA extraction kit (Qiagen, Germany) according to manufacturer recommendation. Extracted RNA was subjected to real time RT-PCR using the LightMix ${ }^{\circledR}$ Modular Hepatitis E Virus (HEV) (TIB MOLBIOL, Germany). Samples in positive serum pools were tested separately to identify the positive individual sample using the same protocol.

\section{Sequencing and phylogenetic analysis:}

Positive individual samples were subjected to nested RT-PCR reaction as described earlier [22, 23]. In brief, a 341 bp fragment of the HEV RNA dependent RNA polymerase (RdRp) gene was amplified and the resulting PCR product was purified using the agarose gel purification kit (Qiagen, Germany) and subjected to direct sequencing using the BigDye dideoxy termination kit on the $A B I 3500$ sequence analyzer (Applied Biosystems, Germany). The full genome sequencing was performed on two positive cases one imported and one domestic case. The full genome sequence was performed according to Lee et al [18]. The full HEV genome was amplified in 19 fragments and the PCR products were purified from agarose gel and sequenced using the same protocol as for the RdRp fragments. The fragments were assembled using the assembly tool of the Geneious prime software (version 2021.12.2) [24]. The generated sequences were multiple aligned using Geneious software and phylogenetic trees were produced using the Neighbor-Joining method with 1000 bootstrap replicates. Sequences were deposited in Genbank and were assigned the accession numbers MZ027568-MZ027584 for the RdRp gene sequences and MW835252-MW835253 for the two full genome sequences.

\section{Results}

\section{Camels and blood sample collection}

Blood samples were collected from 1,189 DCs: 893 (75.1\%) were from DCs imported from Africa (724, from Sudan and 169 from Djibouti). Two hundred and ninety-six were domestic Saudi DCs from local farms and slaughterhouses in and around Jeddah. 1,085 out of 1189 DCs were male (91.3\%) and $104 / 1,189(8.7 \%)$ were female. Nine hundred and fifty two out of $1,189(80.1 \%)$ DCs were $3-5$ years old. $229(19.2 \%)$ were between $1-3$ years of age and $8(0.7 \%)$ were $<1$-year-old.

\section{RT-PCR DCHEV RNA testing}

RT-PCR testing of the samples showed DcHEV RNA in 21/1,189 camels (1.77\%) with a higher prevalence of DcHEV RNA in domestic dromedaries (4.1\%) as compared to the imported dromedaries $(1.0 \%)$. All positive cases were male dromedaries and all the positive imported dromedaries were imported from Sudan. The highest prevalence was found in the juvenile dromedaries $<1$ year $(1 / 8,12.5 \%)$, followed by the age group $1-3$ years $(9 / 229,3.9 \%)$, while the lowest prevalence was found in the age group $3-5$ years $(11 / 952,1.2 \%)$. 


\section{Sequencing and phylogenetic analyses}

Positive PCR samples were subjected to direct sequencing, and we were able to sequence 19 out of 21 positive samples in the RdRp gene. Phylogenetic analysis of the generated sequences showed that all sequences belong to HEV genotype 7. They clustered with sequences reported earlier mainly from UAE and Kenya. The full genome sequences showed clustering with the two available full genome sequences in the Genbank. They showed 242 nucleotide mutations including 24 nonsynonymous mutations that led to amino acid changes along the viral genome when compared to the reference sequence KJ496143-HEV$7 a$.

Figure 1 shows the grouping of all sequencing with the reference sequences of HEV genotype 7 (KJ496143 and KJ496144) together with the previously reported GT-7 sequences by Rasche et al[22]. Phylogenetic tree also shows the clustering of the sequences from domestic camels distant from sequences of imported camels (except for three samples) with nucleotide identity matrixes ranging from 80.4-91.3\%. Domestic sequences also clustered close to sequences reported from Africa (KM820915) and UAE (KM820907-11) (with identity matrixes ranging from 90.7-96.8\%).

Figure 2 shows the clustering of the two full genomes generated from this study with the two available genotype 7 from Genbank.

\section{Discussion}

Our data add further to the growing knowledge on the role of HEV as a possible camel associated zoonotic pathogen with epidemic potential. There are several notable findings from our study.

First, our study of DcHEV in domestic and imported DCs from Africa in Saudi Arabia is the largest published to date and provides evidence for the presence of DcHEV in both domestic and imported camels from Africa with evolving genomic changes.

Second, phylogenetic analysis of the RdRp sequences identified infection with HEV genotype 7. When comparing with HEV genotype 7 sequence KJ496144, the two full genome sequences from our study showed 242 nucleotide variations including 24 nonsynonymous mutations leading to the amino acid changes.

Third, sequences from domestic camels formed a separate cluster together with published sequences from Kenya, Somalia, and UAE.

Fourth, the sequences of imported camels clustered with one DCHEV isolate previously reported from UAE indicating a possible common source of animal infection. Both domestic and imported sequences clustered away from isolates reported from Pakistan.

Fifth, full-genome sequences generated from this study were added to the only two full-genome sequences of genotype 7 in GenBank. The analysis showed a 24 amino acid difference between our 
sequences and the GenBank sequences while the other 218 nucleotide mutations were synonymous mutations and did not result in amino acid changes.

Sixth, we found that domestic DCs from Saudi Arabia had higher prevalence of DcHEV RNA than imported camels from Africa.

$\mathrm{HEV}$ is a cause of morbidity in several regions around the world, where it is endemic in some developing countries with poor sanitation/hygiene and frequent water contamination, which are the most common risk factors [25]. While in developed countries like the US, Japan and Europe, the most common risk factor is the consumption of infected meat and animal products [26]. The zoonosis of HEV was established by the identification and characterization of HEV isolated from pigs that have close genetic relationship to human HEV [27]. Several other reports on the transmission of HEV to human through consumption of animal products or frequent contact with animals have led to the acknowledgement of HEV as an important human zoonosis [5]. HEV studies from Saudi Arabia have been scanty. One seroprevalence study in 2002 reported HEV seroprevalence ranging from ( $7.2 \%$ vs. $10.8 \%)$ in dialysis patients from Jeddah as compared to $16.9 \%$ and $18 \%$ HEV seroprevalence in blood donors in Jeddah (West) [15] and sickle cell anemia in children from Jizan (South) [13, 14], respectively.

The importance of investigating HEV infection in camels in Saudi Arabia comes from the social and economic role that dromedaries play in the Arabian Peninsula. Dromedaries constitute a source of income through camel trade, camel races and camel shows and consumption of meat and milk. Camel herds frequently move across the Arabian Peninsula and might cross the borders of many Gulf countries for many reasons such as grazing, participation in camel races and camel shows. Camels' frequent movement might increase the risk of transmitting the infection to different populations of these countries. However, there is no official national identification procedures nor obligatory vaccination campaigns focused on camels; it is difficult to quantify the extent of these movements. Camels from both UAE and Qatar travel every year during the winter seasons (November - February) for grazing, shows and racing in the Eastern region of Saudi Arabia.

The main source of dromedary camels to the Arabian Peninsula is the importation of life camels from the Horn of Africa (Sudan, Djibouti, Somalia, Ethiopia and Kenya) to the Arabian Peninsula. The importance of performing this study in Jeddah comes from the geographical location of the seaport in Jeddah as the main port of entry of dromedaries from Africa [28]. In 2013, official records in Saudi Arabia reported the import of 131,932 camels representing more than $70 \%$ of the animals slaughtered in the country [29]. Detailed spatiotemporal studies of camel movement patterns are lacking and are urgently needed to try and understand the role of camel movement in spreading the infection.

Recent studies from UAE have also identified the presence of DcHEV-specific viral genomes in DCs where calves became infected during the first 6 months of life and cleared the virus with time [19]. In this study, the prevalence of HEV RNA was higher in domestic camels (4.1\%) compared to imported camels (0.9\%). The difference might be due to the younger age of domestic camels (the majority are 1-3 years old) where they acquire the infection early in life compared to the imported camels (all are 4-5 years old) where they 
have most probably acquired and cleared the DcHEV infection. Alternatively, this higher prevalence might indicate a possible risk factor for the infection in domestic camels responsible for this higher prevalence. In a recent study on the same subset of samples [21], we showed that the seroprevalence of DcHEV in domestic camels is slightly higher than those of imported camels $(25.4 \%$ vs. $22.4 \%$, p value $=0.3)$. Observations from both studies warrant the need for further investigations to identify the possible risk factor that is causing this higher prevalence.

Both domestic and imported sequences clustered away from isolates reported from Pakistan. This indicates the presence of a genetic difference between sequences from this study and those from Pakistan. Further investigation is needed to clarify the significance of these differences. Phylogenetic analysis of the RdRp sequences in this study shows that they belong to HEV genotype 7. Sequences from domestic camels formed a separate cluster with reported sequences from Kenya, Somalia, and UAE (except for three samples) while imported sequences clustered with one isolate from UAE indicating a possible common source of infection. Previous studies have demonstrated the HEV seropositive DCs in Africa and the Middle East [17, 21, 30-33]. A recent study [33] showed a higher seroprevalence in dromedaries in Israel where they found a seroprevalence of $68.6 \%$. Further investigation is needed to identify the clinical role of these amino acid changes in the course of the disease. The duration seropositivity and the protective immune mechanisms also need to be defined.

Our study had several limitations. Whilst our DCs study sample was large, the number of positive DcHEV cases was small. Pooling leads to sample dilution which might have affected prevalence. The prevalence of HEV RNA might be underestimated because lifespan of viraemia in serum is short [19].

Our results provide evidence for the presence of DcHEV in both domestic and imported camels from Africa with evolving genomic changes. Further surveillance studies are required to identify the role of the identified DcHEV amino acid changes in the course of the disease. Our data highlight the potential role of DcHEV in the burden of HEV in the Saudi population specially in populations who have frequent contact with dromedaries like camel handlers and slaughterhouse workers and those who frequently consume camel meat and milk. Any change in transmissibility and virulence of DcHEV in DCs would affect the risks of spread to humans and its potential impact on national and regional public health security. Further clinical, serological, molecular and epidemiology investigations of DcHEV in human populations in the Middle East and Africa are needed to define and monitor the potential threat of DcHEV to Middle eastern and global public health security in light of 10 million pilgrims who visit Saudi Arabia every year from 182 countries.

Study Limitation: The low number of domestic camels compared to imported camels could not allow for statistical significance in the differences between the two groups of camels.

\section{Declarations}

Author declarations: 
All authors have an interest in ONE-HEALTH and zoonotic infections. All authors declare no conflicts of interest

\section{Acknowledgements:}

The work was supported by the King Fahd Medical Research Center. Sir Prof Alimuddin Zumla, is coPrincipal Investigator of the (PANDORA-ID-NET), the Pan-African Network For Rapid Research, Response, Relief and Preparedness for Infectious Disease Epidemics, supported by the EDCTP. He is in receipt of a UK National Institutes of Health Research, Senior Investigator Award and is a Mahathir Foundation Science Award laureate.

\section{Data sharing Statement:}

Data collected for the study, on camels, and molecular analyses can be made available upon request

\section{References}

1. Azhar, E. I. et al. Evidence for Camel-to-Human Transmission of MERS Coronavirus.New England Journal of Medicine2014, 370, (26),2499-2505

2. El-Kafrawy, S. A. et al. Enzootic patterns of Middle East respiratory syndrome coronavirus in imported African and local Arabian dromedary camels: a prospective genomic study. The Lancet. Planetary health, 3 (12), e521-e528 (2019).

3. Memish, Z. A., Perlman, S., Van Kerkhove, M. D. \& Zumla, A. Middle East respiratory syndrome.Lancet (London, England)2020,395, (10229), 1063-1077

4. Ben Abid, F. et al. Middle East respiratory syndrome coronavirus infection profile in Qatar: An 8-year experience. IDCases 2021, 24, e01161

5. Nan, Y., Wu, C., Zhao, Q. \& Zhou, E. M. Zoonotic Hepatitis E Virus: An Ignored Risk for Public Health.Front Microbio/2017, 8,2396

6. Zhang, Y., Gong, W., Zeng, H. \& Wang, L. Genetic Evolution of Hepatitis E Virus. Adv Exp Med Biol, 948, 73-88 (2016).

7. Kamar, N. et al. Hepatitis E virus infection.Nat Rev Dis Primers2017, 3,17086

8. Raj, V. S. et al. Novel hepatitis E virus in ferrets, the Netherlands. Emerging infectious diseases, 18 (8), 1369-1370 (2012).

9. Krog, J. S., Breum, S. O., Jensen, T. H. \& Larsen, L. E. Hepatitis E virus variant in farmed mink, Denmark. Emerging infectious diseases, 19 (12), 2028-30 (2013).

10. Xia, J. et al. Swine and rabbits are the main reservoirs of hepatitis E virus in China: detection of HEV RNA in feces of farmed and wild animals. Arch Viro/2015,160, (11), 2791-8

11. Spahr, C., Knauf-Witzens, T., Vahlenkamp, T., Ulrich, R. G. \& Johne, R. Hepatitis E virus and related viruses in wild, domestic and zoo animals: A review. Zoonoses and public health, 65 (1), 11-29 (2018). 
12. Matsuda, H., Okada, K., Takahashi, K. \& Mishiro, S. Severe hepatitis E virus infection after ingestion of uncooked liver from a wild boar. J Infect Dis, 188 (6), 944 (2003).

13. al-Fawaz, I. et al. Hepatitis E virus infection in patients from Saudi Arabia with sickle cell anaemia and beta-thalassemia major: possible transmission by blood transfusion. J Viral Hepat, 3 (4), 203-5 (1996).

14. Abdelaal, M. et al. Epidemiology of hepatitis E virus in male blood donors in Jeddah, Saudi Arabia. Irish journal of medical science, 167 (2), 94-6 (1998).

15. Ayoola, E. A., Want, M. A., Gadour, M. O., Al-Hazmi, M. H. \& Hamza, M. K. Hepatitis E virus infection in haemodialysis patients: a case-control study in Saudi Arabia. J Med Virol, 66 (3), 329-34 (2002).

16. Johargy, A. K., Mahomed, M. F., Khan, M. M. \& Kabrah, S. Anti hepatitis E virus seropositivity in a group of male blood donors in Makkah, Saudi Arabia. J Pak Med Assoc, 63 (2), 185-9 (2013).

17. Woo, P. C. et al. New hepatitis E virus genotype in camels, the Middle East. Emerging infectious diseases, 20 (6), 1044-8 (2014).

18. Lee, G. H. et al. Chronic Infection With Camelid Hepatitis E Virus in a Liver Transplant Recipient Who Regularly Consumes Camel Meat and Milk. Gastroenterology2016,150, (2), 355-7.e3

19. Corman, V. M. et al. Hepatitis E Virus Genotype 7 RNA and Antibody Kinetics in Naturally Infected Dromedary Calves, United Arab Emirates. Emerging infectious diseases, 26 (9), 2214-2217 (2020).

20. Younan, M., Bornstein, S. \& Gluecks, I. V. MERS and the dromedary camel trade between Africa and the Middle East. Tropical animal health and production2016, 48, (6),1277-82

21. El-Kafrawy, S. A. et al. Seroprevalence of Dromedary Camel HEV in Domestic and Imported Camels from Saudi Arabia. Viruses, 12 (5), 553 (2020).

22. Rasche, A. et al. Hepatitis E Virus Infection in Dromedaries, North and East Africa, United Arab Emirates, and Pakistan, 1983-2015. Emerging infectious diseases, 22 (7), 1249-52 (2016).

23. Drexler, J. F. et al. Bats worldwide carry hepatitis $E$ virus-related viruses that form a putative novel genus within the family Hepeviridae. Journal of virology2012, 86, (17),9134-9147

24. Kearse, M. et al. Geneious Basic: An integrated and extendable desktop software platform for the organization and analysis of sequence data., Vol. 28, 1647-9 (2012).

25. Goel, A. \& Aggarwal, R. Advances in hepatitis E - II: Epidemiology, clinical manifestations, treatment and prevention. Expert Rev Gastroenterol Hepatol, 10 (9), 1065-74 (2016).

26. Dalton, H. R., Hunter, J. G. \& Bendall, R. Autochthonous hepatitis E in developed countries and HEV/HIV coinfection. Semin Liver Dis, 33 (1), 50-61 (2013).

27. Meng, X. J. et al. A novel virus in swine is closely related to the human hepatitis E virus. Proc Natl Acad Sci U S A, 94 (18), 9860-5 (1997).

28. B, H. R. A. F., Typology of camel farming system in Saudi Arabia.Emirates Journal of Food and Agriculture2013, 25, (4),250-160

29. Mackay, I. M. \& Arden, K. E. Middle East respiratory syndrome: An emerging coronavirus infection tracked by the crowd. Virus Res, 202, 60-88 (2015). 
30. Li, T. C. et al. Serological evidence of hepatitis E virus infection in dromedary camels in Ethiopia. Journal of Virological Methods, 246, 34-37 (2017).

31. Obaidat, M. M. \& Roess, A. A. Seroprevalence and risk factors of Hepatitis E infection in Jordan's population: First report. Int J Infect Dis, 66, 121-125 (2018).

32. Sayed, I. M., Elkhawaga, A. A. \& El-Mokhtar, M. A. Circulation of hepatitis E virus (HEV) and/or HEVlike agent in non-mixed dairy farms could represent a potential source of infection for Egyptian people. International journal of food microbiology, 317, 108479 (2019).

33. Bassal, R. et al. Seroprevalence of hepatitis E virus in dromedary camels, Bedouins, Muslim Arabs and Jews in Israel, 2009-2017. Epidemiology and infection, 147, e92 (2019).

\section{Figures}

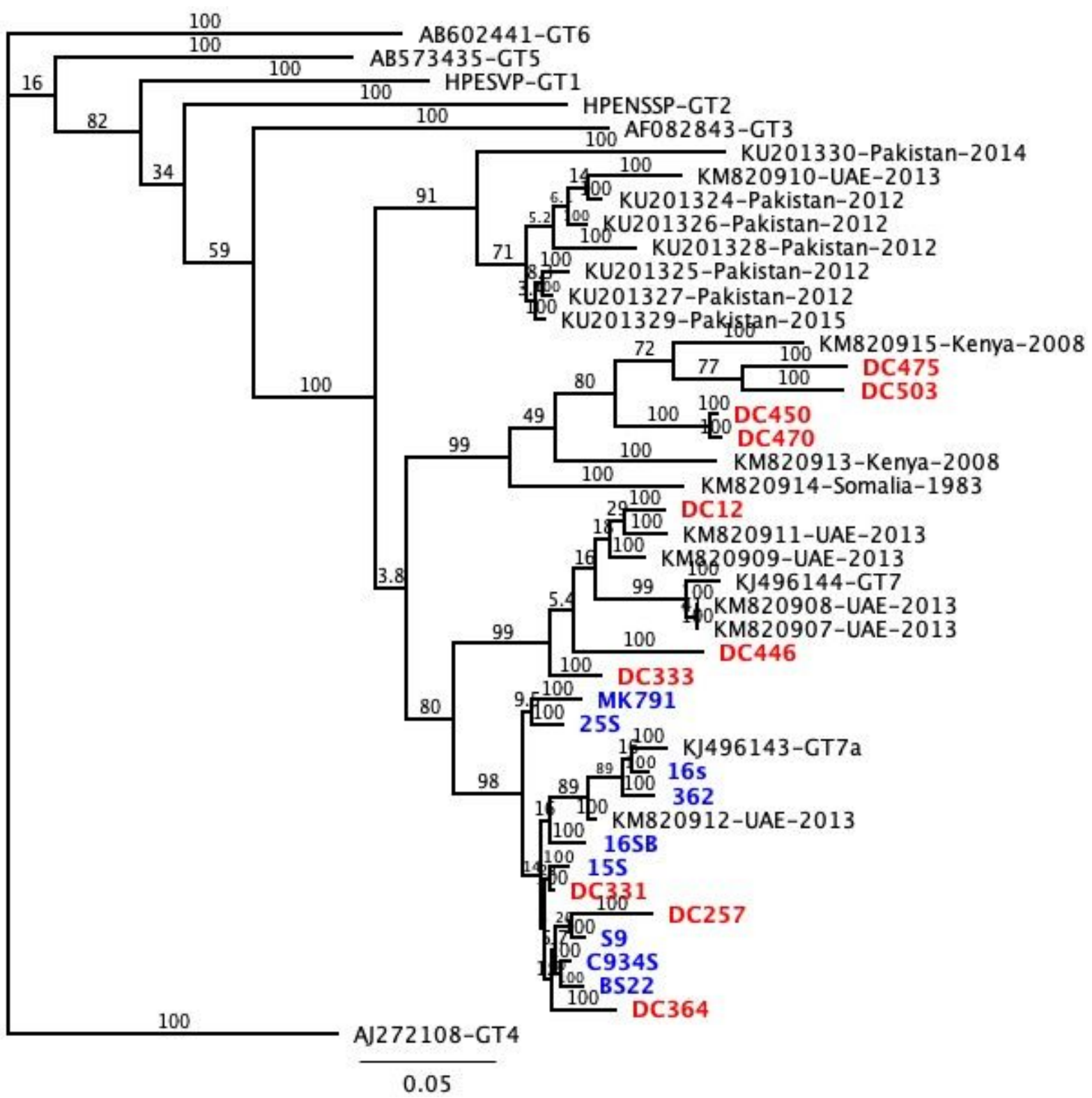

Figure 1 
Phylogenetic tree for the RdRp gene of DcHEV Red-colored sequences represent domestic camels while blue colored sequences represent imported sequences. Phylogenetic tree was constructed using Neighbor-Joining method with 1000 bootstrap replicates after alignment with CLUSTALW using Geneious prime software (version 2021.2.2).

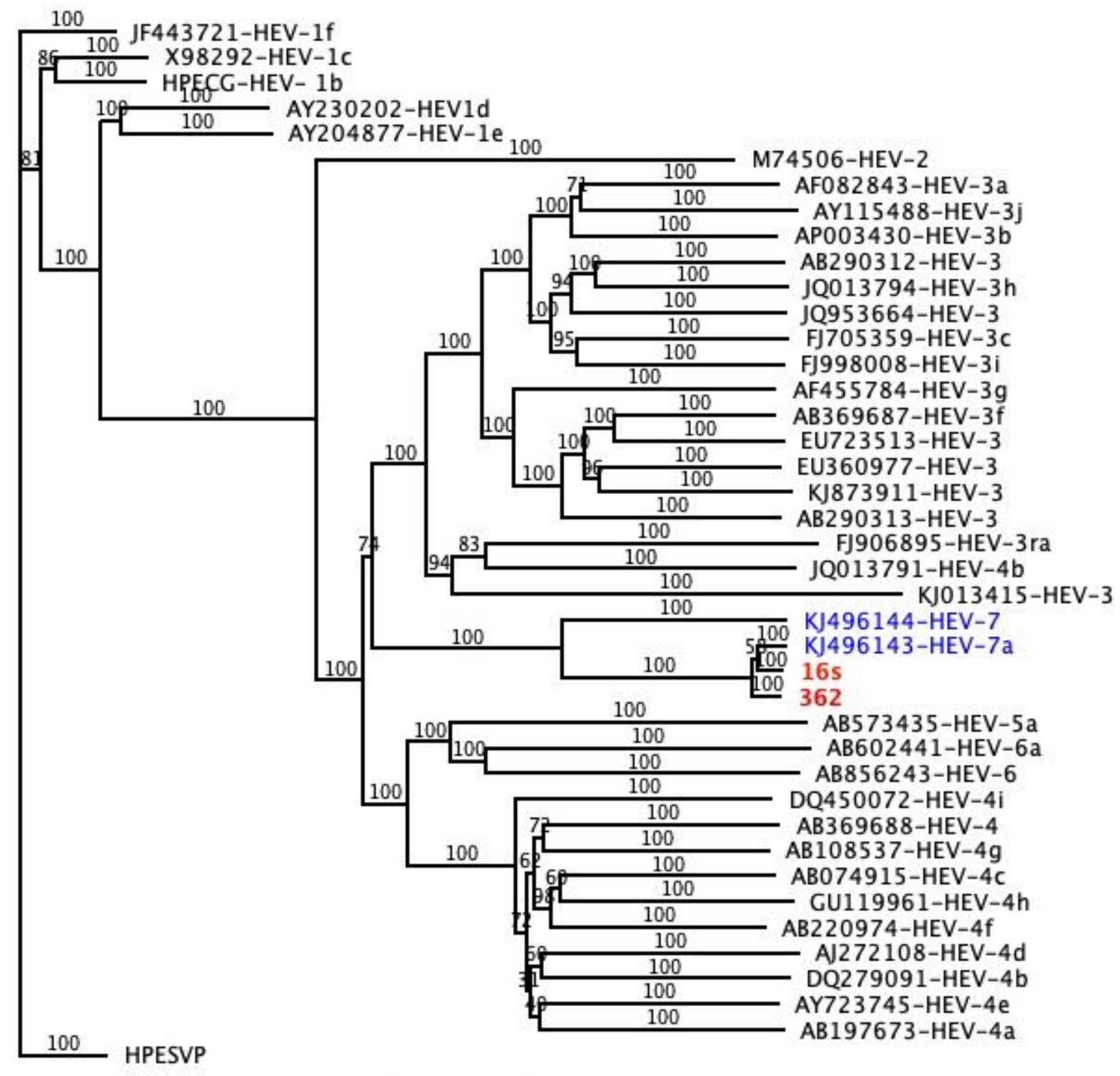

0.05

Figure 2

Phylogenetic tree for the two full genome sequences generated in the study. Red-colored labels represent the new sequences from this study, while the blue-colored labels represent the two full genome reference sequences available in the Genbank. Phylogenetic tree was constructed using Neighbor-Joining method with 1000 bootstrap replicates after alignment with CLUSTALW using Geneious prime software (version 2021.2.2). 